\title{
Patología cardiovascular en pacientes con infección por el virus de inmunodeficiencia humana
}

\author{
Cardiovascular pathology in patients with human immune deficiency
} virus infection

\author{
Germán Valenzuela-Rodríguez ${ }^{1,2,3}$ \\ Médico Cirujano, Doctor en Medicina. \\ ${ }^{2}$ Especialista en Medicina Interna y Cardiología. \\ ${ }^{3}$ Fellow of the American College of Physicians.
}

\begin{abstract}
Resumen
La infección por el virus de inmunodeficiencia humana $(\mathrm{VIH})$ estuvo asociada a un incremento de la morbimortalidad, dado que inducia un estado de inmunosupresión severa, la cual facilitaba la aparición de infecciones oportunistas. Sin embargo, posterior a la aparición y al uso de la terapia antirretroviral de gran actividad (TARGA), los pacientes infectados han experimentado una disminución de mortalidad asociada a infecciones, un incremento de la sobrevida y un incremento de enfermedades cardiovasculares. La etiología de estas enfermedades podría estar relacionada con la infección por si misma, con condiciones proaterogénicas asociadas al empleo de determinados grupos de fármacos antirretrovirales, o con otros eventos adversos que estos serían capaces de inducir individualmente 0 al combinarse con otros medicamentos. Considerando que la mayoria de condiciones asociadas al compromiso cardiovascular en pacientes con $\mathrm{VIH}$ tiene inicio agudo, presentamos esta minuciosa revisión para reconocerlas en las salas de emergencia, eligiendo el tratamiento oportuno y excluyendo los tratamientos inapropiados.
\end{abstract}

Palabras clave: Corazón, HIV, enfermedades cardiovasculares, síndrome de inmunodeficiencia adquirida.

\begin{abstract}
Human immunodeficiency virus (HIV) infection increases both morbidity and mortality by inducing severe immunosupression that generates opportunistic infections. Following use of high active antiretroviral therapy (HAART) in infected patients, infection-related mortality has decreased and both survival and cardiovascular disease have increased. The etiology of cardiovascular disease could be related to either infection itself, proatherogenic conditions associated with antiretroviral therapy or other adverse events caused by these therapies or by combination with other drugs. As most conditions associated with cardiovascular disease have an acute onset, we present this review for their recognition in the emergency ward, choosing opportune treatment and excluding inappropiate ones.

Key words: Heart, HIV, cardiovascular diseases, acquired inmunodeficiency sindrome.
\end{abstract}

An Fac med. 2012;73(4):315-20

\section{INTRODUCCIÓN}

El identificar y tratar la enfermedad cardiovascular en los individuos infectados por el virus de inmunodeficiencia humana (VIH) se ha hecho necesario, debido al compromiso inducido por la propia infección y por las terapias usadas para controlarla. De acuerdo con el Programa de Naciones Unidas conjuntas (Joint United Nations Program), en el año 2010, se ha estimado que 32,8 millones de personas estuvieron infectadas en el mundo. Si bien es cierto, la incidencia anual de nuevos casos de infección está disminuyendo, la prevalencia de la enfermedad cardiovascular está incrementándose por el uso de la terapia antirretroviral de gran actividad (TARGA). Como resultado de los avances en el tratamiento con regímenes complejos de medicamentos y un incremento en la expectativa de vida asociada, las manifestaciones tardías de la enfermedad -como son las enfermedades cardiovasculares y los efectos adversos de la medicación- son cada vez más importantes ${ }^{(1)}$.
Esta infección se caracteriza por un estado profundo de inmunosupresión, que predispone al paciente a infecciones por gérmenes oportunistas, neoplasias malignas y a una disfunción progresiva de diferentes órganos y sistemas ${ }^{(2)}$.

El compromiso cardíaco en pacientes con síndrome de inmunodeficiencia humana adquirida (sida) fue descrito por primera vez, en 1983, por Austran, quien encontró sarcoma de Kaposi miocárdico en una autopsia. Después de ello, se ha encontrado una prevalencia 
Tabla 1. Eventos adversos inducidos por fármacos antirretrovirales.

Fármacos antirretrovirales

Inhibidores nucleósidos de la transcriptasa reversa

- Abacavir

- Didanosina

- Emtricitabina

- Lamivudina

- Estavudina

- Tenofovir

- Zidovudina
- Incremento potencial de eventos cardiovasculares

- Hipertensión, infarto de miocardio

- Hipertrigliceridemia

- Posible cardiotoxicidad en fetos

- Redistribución de grasas, dislipidemia

- Reducción modesta en colesterol total y LDL

- Potencial disfunción mitocondrial/miopatía

Inhibidores no nucléosidos de la transcriptasa reversa

(Muchas interacciones con otros fármacos, como bloqueadores de canales de calcio (BCC), warfarina, betabloqueadores, quinidina, corticosteroides y teofilina)

- Delavirdina

- Efavirenz

- Etravirina

- Nevirapina

Pueden incrementar el intervalo OT del ECG

- Interacciones farmacológicas

- Arritmias fatales por interacciones con antiarrítmicos. Dislipidemia.

- Hipertensión, dislipidemia aterogénica. Interacciones farmacológicas como efecto de clase.

- Interacciones farmacológicas

\section{Inhibidores de proteasa}

(Aterosclerosis prematura, dislipidemia, resistencia a la insulina, diabetes mellitus, adelgazamiento y redistribución de grasa, incremento de niveles de fibrinógeno)

(Interacción con medicamentos metabolizados por grupo CYP: antimicrobianos, antidepresivos, antihistamínicos, cisaprida, estatinas: lovastatina, simvastatina, sildenafilo, teofilina, amiodarona, disopiramida, flecainida, lidocaína, metilxetina, propafenona y quinidina)

- Amprenavir

- Atazanavir

- Darunavir

- Fosamprenavir

- Indinavir

- Lopinavir/ritonavir

- Nelfinavir

- Ritonavir

- Saquinavir

- Tipranavir

\section{Incremento del intervalo PR del ECG}

- Hipercolesterolemia, hipertrigliceridemia, hipotensión (en usuarios de sildenafilo)

- Interacciones con BCCs y bloqueadores del nodo atrioventricular

- Hipotensión y otras interacciones con estatinas y BCCs

- Hiperlipidemia e interacciones farmacológicas

- Incrementa el riesgo de infarto de miocardio. Hipotensión y miopatía por interacción con estatinas y antihipertensivos

- Prolongación del intervalo PR; bloqueo atrioventricular de segundo 0 tercer grado, otras anormalidades de conducción

- Interacciones farmacológicas con estatinas y otros fármacos

- Hiperlipidemia. Hiperglicemia. Si se prescribe con saquinavir puede incrementar los intervalos PR o segmento QT con el riesgo de arritmia

- Hiperlipidemia, anormalidades de conducción cardíaca e interacciones farmacológicas

- Interacciones farmacológicas con otros fármacos cardiovasculares

Inhibidores de integrasa

- Raltegravir

- $\quad$ Aún no reportadas

Inhibidores de entrada y de fusión

- Enfuvirtida

- Maraviroc

- $\quad$ Aún no reportadas

- Algunos casos de angina, insuficiencia cardíaca e infarto de miocardio. Hipotensión postural

Terapias combinadas

- Efavirenz/emcitrabina/tenofovir (Atripla $\left.{ }^{\circledR}\right)$

- $\quad$ Lamivudina/zidovudina (Combivir ${ }^{\circledR}$ )

- Lamivudina/avacavir (Epzicom $\left.{ }^{\circledR}\right)$

- $\quad$ Lamivudina/zidovudina/abacavir (Trizivir ${ }^{\circledR}$ )

- Emcitrabina/tenofovir (Truvada ${ }^{\circledR}$ )

- Interacción con drogas metabolizadas por vía CYP3A4

- Redistribución o acumulación de la grasa corporal, cardiomiopatía. Posible cardiotoxicidad en el feto

- Infarto de miocardio

- Cardiomiopatía, hipotensión

- Aún no reportadas. Evaluar las interacciones de cada medicamento por separado. 
del compromiso cardíaco en pacientes con sida entre 28 y $73 \%$, dependiendo del tipo de estudio realizado. En la infección por el VIH, es frecuente encontrar en las autopsias signos de compromiso cardíaco en más de $73 \%$ de pacientes fallecidos ${ }^{(2)}$.

Al incrementarse la expectativa de vida en la población con infección por el VIH, la incidencia de enfermedades relacionadas con la edad, como es el caso de las enfermedades cardiovasculares, se ha incrementado también. Este incremento ha sido atribuido a la combinación del envejecimiento con el incremento de factores de riesgo cardiovascular, como son la hipertensión y la diabetes, así como con el uso de los medicamentos antirretrovirales cuyos efectos dependerán parcialmente de la clase terapéutica y también de los efectos individuales de cada fármaco ${ }^{(1,2)}$ (ver tabla 1).

Por otro lado, vale la pena destacar que otros fármacos prescritos no por la infección en sí sino por otras comorbilidades, podrían tener interacciones importantes al combinarse con la medicación antirretroviral. Por ello, debemos considerar esas interacciones potenciales al elegir la medicación antirretroviral o al elegir la medicación concomitante en nuestros pacientes (ver tabla 2).

La presente revisión incluye una descripción de las patologías cardiovasculares más frecuentemente asocia- das a la infección por el VIH o a sus terapias, las cuales pueden presentarse como verdaderas emergencias médicas (ver tabla 3).

\section{COMPROMISO ASOCIADO A LA INFECCIÓN POR EL VIH O A SUS TERAPIAS}

\section{A) Compromiso cardiaco}

\section{Compromiso miocárdico}

\subsection{Cardiomiopatía dilatada}

La infección por el VIH es reconocida como una causa importante de cardiomiopatía dilatada, con una prevalencia reportada de 3,6\% entre todos los pacientes con cardiomiopatía. En comparación con los pacientes que tienen cardiomiopatía dilatada idiopática, los pacientes con cardiomiopatía dilatada por esta causa, tienen una supervivencia disminuida y una probabilidad de muerte cuatro veces mayor ${ }^{(2-4)}$.

La importancia de la disfunción cardíaca es demostrada por una mediana de supervivencia de 101 días en pacientes con disfunción ventricular izquierda, comparada con 472 días en aquellos con una función cardíaca normal, para grupos de pacientes en el mismo estadio de infección. La miocarditis representa aún la causa más común de cardiomiopatía dilatada en pacientes con infección por el VIH ${ }^{(2-4)}$.
Además de la contribución de la infección por el VIH en su etiología, se ha propuesto otras variables que podrían tener rol preponderante, como por ejemplo selenio, vitamina B12, carnitina, hormona de crecimiento y hormonas tiroideas. Los síndromes de mala absorción y diarrea pueden condicionar la pérdida de estos elementos que han sido relacionados directa o indirectamente con la presencia de cardiomiopatía ${ }^{(2,4)}$.

La cardiotoxicidad por fármacos en los pacientes infectados por el VIH es controversial, especialmente para el caso de la zidovudina, la cual provocaría una destrucción difusa de las ultraestructuras e inhibición de la replicación del $\mathrm{ADN}$, condicionando acidosis láctica, la misma que contribuye a la disfunción miocárdica. Sin embargo, no se ha encontrado relación con el empleo de los inhibidores de la transcriptasa reversa, aunque otras drogas tendrían un rol importante en los cuadros de toxicidad en esta población, como son la doxorrubicina, interferón alfa, foscarnet, cotrimoxazol, pentamidina y ganciclovir. Algunos agentes tóxicos, como el alcohol y la cocaína, actuarían negativamente sobre el miocardio, agravando la disfunción ventricular establecida ${ }^{(2,4)}$.

\subsection{Miocarditis}

Las miocarditis y las infecciones miocárdicas en los pacientes con infección por el VIH son las causas más estudiadas de

Tabla 2. Interacciones farmacológicas más comunes entre fármacos cardiovasculares y medicamentos antirretrovirales.

\begin{tabular}{|c|c|c|}
\hline Fármaco cardiovascular & Fármacos antirretrovirales & Efecto \\
\hline $\begin{array}{c}\text { Antiarrítmicos } \\
\text { (amiodarona, flecainida) }\end{array}$ & Todos los inhibidores de proteasa & $\begin{array}{l}\text { Incremento de la concentración del antiarrítmico; } \\
\text { se recomienda monitoreo }\end{array}$ \\
\hline Digoxina & Ritonavir & $\begin{array}{l}\text { Incremento de la concentración del antiarrítmico; } \\
\text { se recomienda monitoreo }\end{array}$ \\
\hline Diltiazem & Atazanavir & $\begin{array}{l}\text { Incremento de la concentración de diltiazem; } \\
\text { se sugiere reducir la dosis a la mitad }\end{array}$ \\
\hline Inhibidores de HMG CoA reductasa & Todos los inhibidores de proteasa & $\begin{array}{c}\text { Rabdomiólisis. Se contraindica la lovastatina y } \\
\text { simvastatina. Se puede usar atorvastatina a dosis } \\
\text { reducidas }\end{array}$ \\
\hline Metoprolol & Todos los inhibidores de proteasa & $\begin{array}{l}\text { Incremento de las concentraciones de metoprolol; } \\
\text { se recomienda monitoreo }\end{array}$ \\
\hline Warfarina & $\begin{array}{c}\text { Todos los inhibidores de proteasa y } \\
\text { delavirdina }\end{array}$ & $\begin{array}{l}\text { Incremento de las concentraciones de warfarina; } \\
\text { se recomienda monitoreo }\end{array}$ \\
\hline
\end{tabular}


miocardiopatía dilatada. Los viriones del VIH tipo 1 parecen infectar las células miocárdicas con una distribución en parche, sin asociación directa con la presencia del virus y disfunción de los miocitos. La necrosis de las fibras miocárdicas es generalmente mínima, con infiltrados linfocíticos leves a moderados. No está aún claro, cómo es que el VIH-1 ingresa a los miocitos, los cuales carecen de receptores CD4; sin embargo, las células dendríticas de reservorio pueden tener un rol en la activación multifuncional de citoquinas que contribuyen a un daño tisular progresivo y tardío, como el factor de necrosis tumoral (FNT), IL-1, IL-6 e IL-10. La coinfección con otros virus (usualmente virus cocksackie B3 y citomegalovirus) puede tener rol patogénico preponderante ${ }^{(2,5-7)}$.

\subsection{Disfunción ventricular derecha e hipertensión pulmonar}

La incidencia de hipertensión pulmonar asociada a la infección por el VIH es aproximadamente $1 / 200$, mucho mayor que la encontrada en la población general, que es $1 / 200000$. Su detección está relacionada con infecciones pulmonares, uso de drogas endovenosas, transfusión de factor VIII en pacientes con hemofilia, tromboembolismo venoso, insuficiencia cardíaca y la presencia de HLA-D26 y HLA-DR52 ${ }^{(2,8,9)}$.

Los síntomas y el pronóstico de los pacientes con hipertensión pulmonar varían de acuerdo con la severidad de la hipertensión, encontrándose desde pacientes asintomáticos hasta pacientes en insuficiencia cardíaca avanzada y cor pulmonale. De todos modos, es importante señalar que los pacientes con hipertensión pulmonar asociada a la infección por el VIH tienen un tiempo de supervivencia menor en comparación con aquellos que no tienen esta condición (1,3 años versus 2,6 años) ${ }^{(2,89)}$. Se ha estimado que ocurre en casi el 0,5\% de pacientes hospitalizados y es una causa de compromiso cardíaco severo con cor pulmonale y muerte asociados ${ }^{(2,8-10)}$.

La patogénesis es multifactorial y poco entendida. El VIH puede causar

Tabla 3. Emergencias cardiovasculares asociadas al compromiso cardiovascular en la infección por el VIH-sida.

\begin{tabular}{|c|c|}
\hline Enfermedad cardiovascular & Emergencias cardiovasculares \\
\hline \multicolumn{2}{|l|}{ Cardíacas } \\
\hline 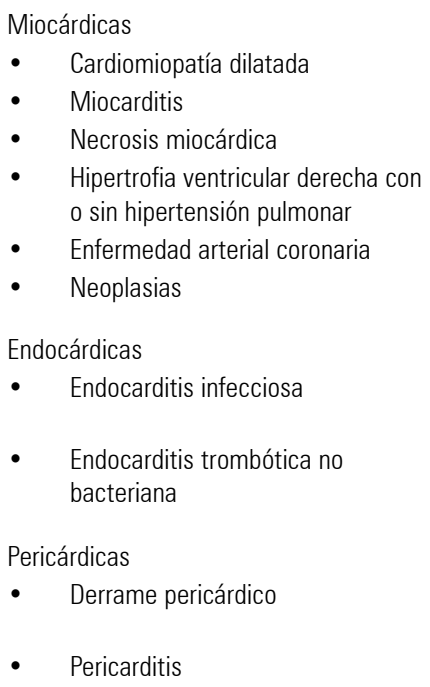 & 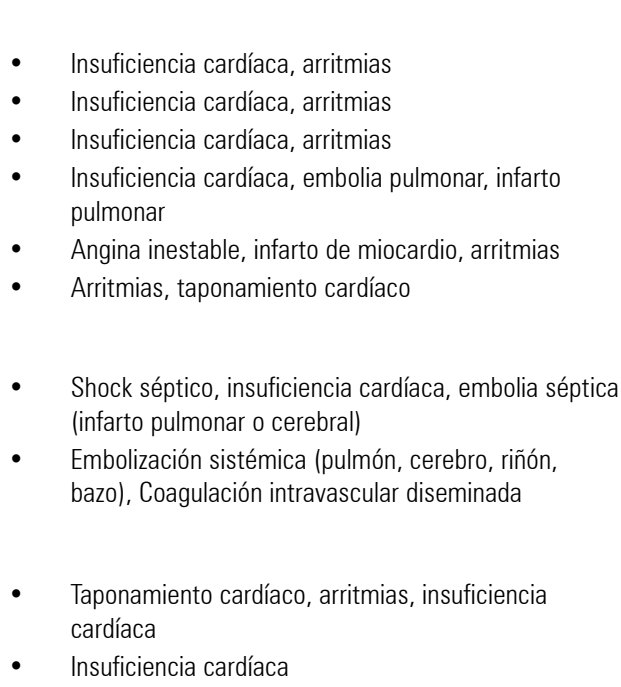 \\
\hline \multicolumn{2}{|l|}{ Extracardíacas } \\
\hline $\begin{array}{ll}\text { - } & \text { Ateroscleróticas } \\
\text { - } & \text { Tromboembólicas } \\
\text { - } & \text { Eventos adversos de medicación o } \\
& \text { interacciones con otros fármacos }\end{array}$ & $\begin{array}{l}\text { - } \\
\text { - } \quad \text { Trombosidentes cerebrovasculares } \\
\text { - } \\
\text { Arritmias, incremento de factores de riesgo } \\
\text { cardiovascular (enfermedad arterial coronaria o } \\
\text { condiciones ateroscleróticas extracardíacas) }\end{array}$ \\
\hline
\end{tabular}

Modificado de ${ }^{(28)}$.

daño endotelial y vasoconstricción relacionada con mediadores, por la estimulación de la glicoproteína 120 de la envoltura, incluyendo la liberación directa de algunas sustancias vasoconstrictoras, como ET-1, IL-6 y FNT ${ }^{(2,8,11)}$.

\subsection{Infarto agudo de miocardio}

La asociación entre la infección por el VIH o por otros virus y las lesiones arteriales coronarias no está aún clara. Las secuencias de VIH-1 han sido recientemente detectadas por el método de hibridación in-situ en los vasos coronarios de un paciente con infección por el VIH que falleció de un infarto agudo de miocardio. Los mecanismos potenciales por los cuales el VIH-1 puede dañar las arterias coronarias incluyen la activación de citoquinas y moléculas de adhesión, así como la alteración de las moléculas de histocompatibilidad clase 1 en la superficie de las células musculares lisas $(2,12-17)$.
En la última década, se ha informado casos de síndromes coronarios agudos (angina inestable o infarto de miocardio) en pacientes con infección por el VIH que reciben antirretrovirales, predominantemente del tipo inhibidores de proteasa. Los pacientes con factores de riesgo adicionales - como hipertensión, diabetes, tabaquismo e hiperhomocisteinemia- pueden tener un riesgo mayor para desarrollar síndromes coronarios agudos o infartos cerebrales, por la acelerada aterosclerosis que en ellos se produce ${ }^{(2,14,17-22)}$.

\subsection{Neoplasias cardíacas}

La prevalencia de sarcoma de Kaposi (SK) cardíaco en pacientes con infección por el VIH en estadio sida varía entre 12 y $28 \%$ en estudios de autopsias, en la era anterior al uso de TARGA. El compromiso cardíaco por SK ocurre generalmente cuando hay un compromiso visceral sistémico ${ }^{(2,23-25)}$. 
Las lesiones son típicamente menores de $1 \mathrm{~cm}$ de diámetro y pueden ser pericárdicas o miocárdicas y, en casos muy raros, estarían relacionadas con obstrucciones al tracto de salida sanguíneo, disfunción miocárdica, mortalidad o morbilidad ${ }^{(2,25)}$.

Los linfomas no Hodgkin (LNH) cardíacos son infrecuentes en el sida. Las lesiones aparecen groseramente o más discretamente de forma nodular, o inclusive de aspecto polipoideo, afectando generalmente al pericardio y más raramente al miocardio. Casi no se acompañan de inflamación ni de necrosis. La mayoría de linfomas son los de células B de grado alto o linfomas tipo Burkitt ${ }^{(2,25)}$.

La introducción de la TARGA ha reducido de manera importante la incidencia del compromiso cardíaco por estas neoplasias en particular, probablemente por la mejoría del estado inmunológico o por la supresión de las infecciones por agentes oportunistas como el virus herpes tipo 8 y el virus Epstein Barr, que tienen rol etiológico preponderante en la aparición de este tipo de neoplasias ${ }^{(2,25)}$.

\section{Compromiso endocárdico}

\subsection{Endocarditis infecciosa}

La prevalencia de endocarditis infecciosa en pacientes infectados por el VIH es similar a la de aquellos pacientes de otros grupos de riesgo, como los usuarios de drogas intravenosas. Esta prevalencia varía entre $6,3 \%$ y $34 \%$ en pacientes infectados por el VIH y que usan drogas intravenosas, independientemente de los esquemas de terapia antirretroviral recibidos. La endocarditis del lado derecho del corazón es la más común, siendo los agentes más frecuentemente aislados el Staphylococcus aureus ( $>75 \%$ de casos), Haemophilus influenzae, Candida albicans, Aspergillus fumigatus y Cryptococcus neoformans. Se encuentra que las vegetaciones son más grandes y de aspecto más friable, con destrucción valvular inclusive ${ }^{(2,5,26-28)}$.

En la evaluación microscópica se encuentra un gran número de neutrófilos, mezclados con colonias de bacterias, plaquetas y fibrina. Estas vegetaciones producen frecuentemente émbolos sépticos. La presentación y supervivencia (85 versus 93\%) de los pacientes con endocarditis infecciosa es similar a la de los pacientes no infectados por el VIH. Sin embargo, los pacientes en estadio avanzado de enfermedad tienen una mortalidad mayor del 30\%, comparándose con aquellos en estadios precoces, la cual podría estar relacionada al grado de inmunosupresión ${ }^{(2,5,26-28)}$.

\subsection{Endocarditis trombótica no bacteriana}

La endocarditis trombótica no bacteriana (endocarditis marántica) ocurre entre 3 y $5 \%$ de pacientes en estadio sida, frecuentemente en aquellos con síndrome de consumo. Las vegetaciones endocárdicas friables son usualmente menores de 0,5 cm y están compuestas de plaquetas, fibrina y pocas células inflamatorias. Estas afectan predominantemente el lado izquierdo del corazón y son una causa poco frecuente de embolización sistémica ${ }^{(2,26,28)}$.

\section{Compromiso pericárdico}

El compromiso pericárdico es una de las formas más comunes de compromiso cardiovascular en la infección por el VIH, existiendo una gran variedad de manifestaciones clínicas, dentro de las cuales se incluyen derrame pericárdico asintomático, pericarditis, taponamiento cardíaco y pericarditis constrictiva.

La prevalencia de derrame pericárdico en pacientes infectados por el VIH oscila entre 21 y $30 \%$, con una incidencia anual en los años anteriores al advenimiento de la TARGA. Es por ello que, en la evaluación de un derrame pericárdico, debe considerarse en la actualidad el despistaje de la infección por el VIH ${ }^{(2,5,11,25,29,30)}$.

\subsection{Derrame pericárdico}

El derrame pericárdico en la infección por el VIH puede estar relacionado a infecciones por gérmenes oportunistas o a neoplasias malignas, pero más frecuentemente a una etiología que no puede precisarse bien. Por otro lado, este hallazgo puede formar parte de un compromiso seroso generalizado que involucra también a las superficies pleurales y peritoneales llamado 'síndrome de fuga capilar', el cual está ampliamente relacionado con la expresión de citoquinas en los estadios finales de enfermedad por VIH. Sin embargo, el derrame pericárdico puede resolverse espontáneamente en más de $42 \%$ de los pacientes ${ }^{(2,30)}$.

Los pacientes en estadio sida que presentan derrame pericárdico tienen una incidencia anual de taponamiento cardíaco de $9 \%$. Debe considerarse también que $1 \%$ de todos los pacientes con sida desarrolla esta condición anualmente, lo cual resalta la importancia de evaluar a este grupo de pacientes, con énfasis en aquellos grupos en estadios avanzados de infección ${ }^{(2,29,30)}$.

Hasta ahora no existe consenso sobre la etiología del derrame pericárdico en la infección por el VIH. Un origen infeccioso pudiera ser el más frecuente, con amplias variaciones geográficas, pero diversos estudios -que incluyen pericardiocentesis, biopsia pericárdica y cultivo- mostraron resultados no concluyentes, por lo cual su utilidad deberá analizarse de acuerdo al contexto clínico de cada paciente ${ }^{(2,30)}$.

\section{B) Compromiso vascular extracardíaco}

\section{Compromiso aterosclerótico vascular}

\subsection{Desórdenes cerebrovasculares}

Además del incremento de la enfermedad cardiovascular, la incidencia de la enfermedad cerebrovascular se ha incrementado en la población con infección por VIH, probablemente por una combinación del envejecimiento de la población y el uso de regímenes de TARGA basados en inhibidores de proteasa, los cuales producen perfiles lipídicos asociados con aterosclerosis generalizada, siendo también la infección por el VIH un factor de riesgo independiente para enfermedad cerebrovascular. Un estudio indicó que el riesgo relativo ajustado para accidente 
cerebrovascular isquémico es de 9,1 y para hemorragia intracerebral de 12,7 , en comparación con la población sin infección por VIH. Por esta razón, en los pacientes con infección por VIH con nuevos signos neurológicos focales, debe considerarse la enfermedad cerebrovascular dentro del diagnóstico diferencial. La evaluación topográfica cerebral sin contraste, considerando si fuera necesario un estudio contrastado a posteriori, permitirá evaluar las lesiones estructurales, recomendándose si fuera el caso, la consulta con un neurólogo ${ }^{(2,3,31-33)}$.

El infarto cerebral y la hemorragia cerebral como una manifestación de la infección por el VIH han sido aún poco estudiados. Las series más recientes describen una mayor proporción de casos con infarto cerebral (de 90 a 95\% de casos) versus hemorragia cerebral (de 5 a 10\% de los casos). En los casos de infartos cerebrales, es posible encontrar una causa en la mayoría de los pacientes, donde la coagulopatía es la más común. Se ha descrito deficiencia de proteína $\mathrm{S}$, niveles incrementados de anticuerpos antifosfolipídicos tipo $\mathrm{G}$ y anticuerpos anticardiolipina. La mayoría de los pacientes de estas series tuvo niveles de linfocitos CD4 menores a $500 \mathrm{cel} / \mathrm{uL}^{(2,32)}$.

\section{Compromiso tromboembólico}

\subsection{Trombosis venosa profunda}

Los pacientes con infección por el VIH tienen una incidencia dos a diez veces mayor de enfermedad tromboembólica que la población general, tanto en los territorios arteriales como venosos. Los médicos en la emergencia deberán tener una alta sospecha para considerar este diagnóstico, en pacientes con signos y síntomas sugestivos de enfermedad tromboembólica. La patogenia es múltiple y puede estar relacionada con desórdenes de la coagulación, como incremento de los niveles de fibrinógeno, dímero $\mathrm{D}$, inhibidor del plasminógeno tisular tipo 1 , antígeno activador del plasminógeno, o una deficiencia de proteína $S^{(2,31-33)}$.

\section{REFERENCIAS BIBLIOGRÁFICAS}

1. Fisher SD, Kanda BS, Miller TL, Lipshultz SE. Cardiovascular disease and therapeutic drug-related cardiovascular consequences in HIV-infected patients. Am J Cardiovasc Drugs. 2011;11(6):38394.

2. Valenzuela G. Enfermedad cardiovascular asociada a la infección por el virus de inmunodeficiencia humana. Panorama mundial y contribuciones peruanas. Rev Soc Per Med Intern. 2005;18(2):23-30.

3. Venkat A, Piontkowsky DM, Cooney RR, Sirvastava AK, Suares GA, Heidelberger CP. Care of the HIVpositive patient in the emergency department in the era of highly active antiretroviral therapy. Ann Emerg Med. 2008;52(3):274-85.

4. Sundstrom JB, Ansori AA. Pathogenesis of AIDSrelated cardiomyopathy. AIDS Rev. 2001;3:36-43.

5. Mishra RK. Cardiac emergencies in patients with HIV. Emerg Med Clin N Am. 2010;28:273-82.

6. Kearney MT, Cottom JM, Richardson PJ, Shah AM. Viral myocarditis and dilated cariomyopathy: mechanisms, manifestations and management. Postgrad Med J. 2001;77:4-10.

7. Mc Carthy RE 3rd, Boehmer JP, Hruban RH, Hutchins GM, Kasper EK, Hare JM, Baughman KL. Long-term outcome of fulminant myocarditis as compared with acute (non fulminant) myocarditis. N Engl J Med. 2000;342(10):690-5.

8. Golpe R, Fernandez-Infante B, Fernandez Rozas S. Primary pulmonary hypertension associated with human immunodeficiency virusinfection. Postgrad Med J. 1998;74:400-4.

9. Pellicelli AM, Barbaro G, Palmieri F, Girardi E, D'Ambrosio C, et al. Primary pulmonary hypertension in HIV disease: a systematic review. Angiology. 2001;52(1):31-41.

10. Pellicelli AM, Palmieri F, D'Ambrosio C, Rianda A Boumis E, Girardi E, et al. Role of human immunodeficiency virus primary pulmonary hypertension. Case reports. Angiology. 1998;49(12):1005-11.

11. Lekakis K, Ikonomidis I. Cardiovascular complications of AIDS. Curr Opin Crit Care. 2010;16:40812.

12. Hsue PY, Waters DD. What a cardiologist needs to know about patients with human inmunodeficiency virus infection. Circulation. 2005;112:3947-57.

13. Opravil M, Pechere M, Speich R, Joller-Jemelka HI, Jenni R, et al. HIV-associated primary pulmonary hypertension. A case-control study. Swiss HIV Cohort Study. Am J Respir Crit Care. 1997;155(3):900-5.

14. Calza L, Manfredi R, Verucchi G. Myocardial infarction risk in HIV-infected patients: epidemiology, pathogenesis, and clinical management. AIDS. 2010;24;789-802.

15. Lobasco G, Tinoco E, Martins L, Ribiero Mo. Doppler echocardiographic evaluation of HIV-positive patients in different stages of the disease. Arq Bras Cardiol. 1999;73:163-8.

16. Grant A, de Cock K. HIV infection and AIDS in the developing world. Br Med J. 2001;322(7300):1475-8.

17. Murphy E, Coller A, Kalish L, Assmann S, Para M, et al. High active antiretroviral therapy decreases mortality and morbidity in patients with advanced HIV disease. Ann Intern Med. 2001;135:17-26.

18. Meng Q, Lima JAC, Lai H, Vlahov D, Celentano D. Coronary artery calcification, atherogenic lipid changes and increased erythrocyte with human immunodeficiency virus-1 treated with protease inhibitors. Am Heart J. 2002;144:642-8.
19. Rhew D, Bernal M, Aguilar D, lloeje U, Blawell M. Association between protease inhibitor use and increased cardiovascular risk in patients infected with human immunodeficiency virus: a systematic review. Clin Infect Dis. 2003;37:959-72.

20. Kuritzkes DR, Currier J. Cardiovascular risk factors and antiretroviral therapy. N Engl J Med. 2003;348(8):679-80

21. Matezky S, Domingo M, Kar S, Noc M, Shah PK, et al. Acute myocardial infarction in human immunodeficiency virus-infected patients. Arch Intern Med. 2003;163(4):457-60.

22. Morrow DA, Antman EM, Charlesworth A, Cairns R, Murphy SA, et al. TIMI risk score for ST-elevation myocardial infarction: a convenient, bedside, clinical score for risk assessment at presentation. Circulation. 2000;102(17):2031-7.

23. Holmberg SD, Moorman AC, Williamson JM, Tong TC, Ward DJ, et al. Protease inhibitors and cardiovascular outcomes in patients with HIV-1. Lancet. 2002;360(9347):1747-8.

24. Klein D, Hurley LB, Quesenberry CP, Sidney S. Do protease inhibitors increased the risk for coronary heart disease in patients with HIV-1 infection?. J Acquir Immune Defic Syndr. 2002;30(5):471-7.

25. Barbaro G, Fisher SD, Pellicelli AM, Lipshultz SE. The expanding role of the cardiologist in the care of HIV infected patients. Heart. 2001;86:365-7.

26. Marco CA, Rothman RE. HIV infection and complications in emergency medicine. Emerg Med Clin N Am. 2008;(26):367-87.

27. Moss R, Munt B. Infection drug use and right sided endocarditis. Heart. 2003;89:577-81.

28. Barbaro G. HIV-associated cardiovascular complications: a new challenge for emergency physicians. Am J Emerg Med. 2001;19:566-74.

29. Silva-Cardoso J, Moura B, Martins L, Mota-Miranda A, Rocha-Gonçalves F, Lecour H. Pericardial involvement in human immunodeficiency virus infection. Chest. 1999;115:418-22.

30. Gouny P, Lancelin C, Girard PM, Hocquet-Cheyne C, Rozembaum W, Nussaume O. Pericardial efussion and AIDS : benefits of surgical drainage. Eur $J$ Cardiothorac Surg. 1998;13(2):165-9.

31. Barbaro G. Cardiovascular manifestations of HIV infection. Circulation. 2002;106:1420-5.

32. Barbaro G, Fisher SD; Lipshultz SE. Pathogenesis of HIV-associated cardiovascular complications. Lancet Infectious Diseases 2001;1:115-124

33. Barbaro G, Klatt EC. HIV infection and the cardiovascular system. AIDS Rev. 2002;4:93-103.

Articulo recibido el 5 de diciembre de 2011 y aceptado para publicación el 15 de julio de 2012.

Conflicto de intereses:

Ninguno.

\section{Correspondencia:}

Dr. Germán Valenzuela Rodriguez

Clínica Médica Cayetano Heredia

Correo electrónico: german.v.valenzuela@gmail.com 